\title{
¿Teoría o metateoría? En el dominio usuario
}

\author{
Yohannis Martin-Lahera \\ Profesora de la Faculdad de Comunicación, Universidad de la Habana. \\ Maestrante en bibliotecología y ciencias de la información. \\ Coordinadora de la especialidad de bibliotecología \\ y ciencias de la información en el Programa de \\ Universialización de la Enseñanza Superior en Cuba. \\ E-mail: yohannis@fcom.uh.cu
}

\section{Resumen}

Se expone en un primer acercamiento cuáles teorías o metateorías han influido en el dominio usuario de la información. Se parte de la relación usuario - sistemas de información para luego analizar algunas cuestiones teóricas, filosóficas e históricas de la noción usuario y sus términos relacionados. Se exponen, además, algunas teorías y concepciones que dominaron y dominan al estudiar al usuario.

\section{Palabras clabe}

Estudo de usuario de la información; Metateoría; Relación usuario-sistemas de información; Teoría.

\section{Theory or metatheory? In user domain}

\begin{abstract}
The theories and metatheories that have had influence on user domain are presented. First, the relationship between user and information systems is commented. Theorical, philosophical and historical issues associated to user notion and related terms are analysed. Some dominant theories and conceptions in user studies are mentioned.
\end{abstract}

\section{Keywords}

Study of the information user; Metatheory; User and systems information relationship; Theory.

\section{USUARIO Y SISTEMAS DE INFORMACIÓN}

Los usuarios en la actualidad constituyen la cuestión medular de toda organización formal. La articulación de sus funciones, estructuras, componentes y estrategias está orientado hacia cómo satisfacer al beneficiario de sus productos (bienes y servicios). El usuario de la organización formal es quién garantiza la continuidad de su existencia.

Las palabras anteriores se pueden ajustar a una gran diversidad de sistemas. No obstante, especial atención se dará en este trabajo a las organizaciones de información y a los usuarios de la información.

Las organizaciones de información en tanto sistemas de información y comunicación tienen entradas y producen salidas esencialmente de carácter informativo que pueden ser, o están constituidas por bienes que se obtienen a través de, los servicios de búsqueda y recuperación que ellas instrumentan.

Su rol de facilitadoras de acceso a la información es evaluado en la actualidad en función de los criterios perceptivos de sus usuarios, ahí reside el rol protagónico que se le confiere al sujeto. Ese protagonismo que se le ha reconocido al usuario data de varias décadas, lo cual se demuestra en los estudios realizados sobre la producción intelectual en ese terreno en 1964 y 1965 por Menzel (1966: 7) y por Castillo (2002).

La elaboración de los discursos teóricos donde se menciona al usuario de información aumentan cada día, sin embargo se considera que queda un largo camino por recorrer en la construcción de teorías sustentoras de los aportes generados en esa dimensión (Núñez Paula, 2003: 27).

Los estudios de usuarios de la información son una de las dimensiones que más ha sido tratada en la producción intelectual de los profesionales de la información. "La evolución de estos estudios ha estado influida por autores tan prestigiosos como Gross y Gross (1927), que fueron pioneros en el empleo de técnicas bibliométricas para la selección y adquisición de fuentes documentales en una biblioteca. (...) También se destacó Fussler (1949), Garfield (1955), Kessler (1963), Price (1963), Goffman y Morris (1970), Brookes (1971), entre otros" (Castillo, 2002).

Los autores examinados plantean que los primeros estudios de usuario se realizaron en la segunda década del siglo 
XX. Ello trajo consigo una preocupación recurrente durante la realización de esta investigación ipor qué empezar a estudiar al individuo que interactúa con los sistemas de información precisamente en la década del 20 siglo XX?

Otra de las cuestiones que emerge con carácter inquisitivo fue qué teorías, concepciones dominaron y dominan al investigar al usuario. Quizás en este pequeño acercamiento no queden esas interrogantes totalmente aclaradas pero al menos se pretende exponer algunos aspectos que permitan decir sobre otras formulaciones además de la comentada y documentada influencia que ha ejercido el conductismo y cognitivismo en la comprensión y estudio del usuario de los sistemas de información, aunque innegable es la importancia y la función matricial que han ejercido estas corrientes en los estudios relativos a los humanos.

\section{USUARIO DE INFORMACIÓN Y SUS TÉRMINOS EQUIVALENTES: ALGUNAS CUESTIONES TEÓRICAS, FILOSÓFICAS E HISTÓRICAS}

Qué entender por usuarios de la información es uno de los primeros imperativos a dilucidar. Las concepciones contemporáneas plantean que usuarios son todos los individuos que utilizan las facilidades que ofrecen los sistemas de información.

Para Alonso (1999) es "aquel individuo que necesita información para el desarrollo continuo de sus actividades, ya sean profesionales o privadas, y que como tal utiliza un servicio o hace uso de un producto informativo". Siguiendo esta definición bajo esta categoría están comprendidos desde los actores del entorno del sistema de información hasta los miembros de la organización. Es decir usuarios de la información son todos y cada uno de los individuos del universo social.

Hasta este punto del análisis conceptual de la categoría usuario parece existir cierto consenso en que los usuarios de la información deben cumplir los siguientes requisitos:

* Tener necesidad de información, y

* ser beneficiario real o potencial de al menos un producto informativo, ya sea un bien o servicio, ofrecido por sistemas de información.

Cómo en las disciplinas informativas se llegó a esta afirmación aparece como interrogante. Para ello resulta fructífero recorrer las distintas definiciones que se han elaborado a través del tiempo.
El punto donde se complejiza la conceptualización del usuario de información para Alonso (1999) es a partir de la utilización de términos sinónimos y las subsecuentes taxonomías derivadas de ellos. Los términos utilizados han sido receptor, destinatario, lector, consumidor, usuario/cliente.

Sin ánimo de agotar o abordar con exhaustiva profundidad él por qué y el cuándo se utilizan cada uno de los términos mencionados con anterioridad, a continuación se presentarán algunos elementos referidos a esos asuntos.

Bajo el influjo de los enfoques de las teorías de la comunicación se comienza a utilizar los términos receptor y destinatario. A pesar que el área usuarios de la información aparece en conexión directa con la Comunicación científica, en ella influyen las mismas concepciones dominantes en las investigaciones en Comunicación de Masas.

La teoría hipodérmica (conocida también como la aguja hipodérmica) en el campo de la Comunicación posee como concepto central sociedad de masas. Esta noción inserta la interpretación de los conglomerados humanos a partir del supuesto que las masas son amorfas y están atomizadas. "La sociedad, caracterizada por el aislamiento psicológico y la impersonalización” (Medina, 1995: 6).

Esta concepción logra su traducción en el campo informacional. Tal y como expresara Linares (2003: 35) "esa neutralidad aparente resulta de particular importancia en la construcción teórica del usuario como objeto (sujeto) de estudio".

La teoría conductista ejerce una gran influencia en las formulaciones generadas a partir de la teoría hipodérmica donde se presenta a un emisor omnipresente y de inagotable capacidad manipuladora. A su vez estos enfoques psicológico y comunicacional ejercen un efecto en el campo informacional.

Al analizar la concepción dominante del polo no-emisor en los estudios comunicacionales e informacionales se puede observar que en ambos se le concede papel activo al emisor y esa dinámica es exclusiva de ese rol; quedando el papel pasivo, despersonalizado conferido al receptor.

Aplicado a las disciplinas informacionales los emisores son los sistemas de información (medios de comunicación en la Comunicación) y los usuarios de información (audiencia en la Comunicación) constituyen los receptores. La mirada al usuario o grupos de estos es solo para advertir qué usaban, qué leían, y es una mirada permeada por el paradigma físico que busca la eficacia a partir de la calidad del funcionamiento técnico de procesos y tecnologías. 
Todo parece indicar que estaba presente la creencia que si se logra hacer funcionar al sistema - como los trabajadores de la información piensan que debe ser -, entonces el usuario podrá obtener la información que busca. Lo cual muestra la idea que el usuario es quién debe adaptarse a los mecanismos que posee el sistema, diseñados a partir de lo que indica la lógica y la tradición de quienes lo organizan y hacen funcionar.

No resulta difícil suponer, entonces, que esta cosmovisión de la categoría usuario en una y otra disciplina responde a las concepciones paradigmáticas imperantes positivismo, pragmatismo y empirismo, además de la impronta conductista y de las implicaciones conceptuales de la noción "sociedad de masas".

El reflejo de esas ideas se trasluce al observar las unidades de análisis en los estudios de usuarios de la información y las técnicas y métodos empleados. Las técnicas son principalmente las encuestas y "se construye al usuario a partir de variables neutrales" (Linares, 2003: 35). Sobre este aspecto se comentará en el próximo acápite.

Si se tomara algún estudio de usuarios de sistemas de información que responde a los esquemas que pautaron las investigaciones hasta los años los 60 se podrá determinar que sus características revelan los siguientes supuestos (Fernández Molina, Moya-Aregón, 2002):

* Las necesidades de información son algo estable e invariable

* El proceso de búsqueda es determinista, no es dinámico ni iterativo

* La no intervención de elementos emocionales, afectivos o psíquicos en el proceso de búsqueda.

Es precisamente en las variables que se toman para construir al usuario donde años más tarde surge el debate (Linares, 2003). Si antes se intentaba construir al sujeto a partir de variables sociodemográficas (profesión, tipo de actividad desempeñada, sector de la actividad, entre otras), a ella se suman variable sociopsicológicas, afectivas, emocionales y cognitivas (actitud, valores, comportamiento, experiencia cognitiva, habilidades cognitivas, etc.).

Una de las manifestaciones de la influencia cognitivista en las disciplinas informativas es la introducción por Belkin (1978) de la premisa estado anómalo del conocimiento para hacer alusión al estado que experimenta el individuo cuando se siente movido hacia la búsqueda de información.
La perspectiva cognitivista luego es criticada y aparece en la segunda mitad de los años 80 Hjorland con su propuesta sociocognitivista de unir el par individuo/ interno con contexto/externo para estudiar y entender al usuario de la información.

En el derrotero para alcanzar el entendimiento del concepto usuario se desarrollan una serie de taxonomías utilizando diferentes criterios que permiten distinguir grupos de usuarios. Dentro de ellas existe el par usuario real y usuario potencial.

El utilizar la clasificación dicotómica potencial- real del usuario supone una verdadera revolución en la concepción del usuario. Hasta ese momento una de las grandes coincidencias de los autores era uso como elemento distintivo del usuario. La clase potencial elimina ese nexo estrecho con el consumo o utilización de la información al establecer que estos son todos los que podrían beneficiarse de los productos del sistema de información y por tanto son los que se toman en cuenta al diseñar nuevos sistemas/ productos o al introducir cambios en ellos.

Ello sugiere la influencia de los enfoques mercadológicos en las disciplinas informativas, donde se comienza a estructurar la organización y funcionamiento de las organizaciones de información a partir de los usuarios.

\section{ESTUDIOS Y FORMACIÓN DE USUARIOS: INFLUENCIAS INTELECTUALES EN SU TEORÍA Y PRÁCTICA INVESTIGATIVA}

Uno de los primeros estudios donde se pretende explorar la evolución de los estudios de usuarios de información fue el realizado por Menzel (1966). En este trabajo el autor investiga los conceptos relacionados con este tipo de estudio, además de la influencia ejercida por los métodos de investigación procedente de otras áreas y los temas abordados en las pesquisas. Otro de los estudios realizados con este fin es el de Siatri (1999).

A pesar de que algunos autores (Sanz,1994) le conceden al segundo decenio del siglo XX la maternidad de los estudios de usuario, otros (Siatri, 1999) se lo adjudican a la década del 40.

La principal razón que expone Siatri como móvil de su planteamiento es la presentación e impacto de los trabajos de Bernal y Urquhart en 1948 durante la conferencia sobre información científica auspiciada por la Royal Society. La Tabla 1 sintetiza los resultados y propósitos de ambos estudios. 


\section{Objetivos y resultados de los estudios Urquhart y Bernal}

\begin{tabular}{|c|c|c|}
\hline & Urquhart & Bernal \\
\hline Resultados & $\begin{array}{l}\text { Conocer: } \\
\text { - Grado de utilidad del ítem prestado en relación } \\
\text { con el año de publicación y soporte. } \\
\text {-Tipos documentos prestados. } \\
\text { - Propósitos del usuario al realizar la consulta de la fuente. }\end{array}$ & $\begin{array}{l}\text { Proveer un perfil general de su muestra, con la limitación } \\
\text { que este autor solo examinó lo relativo a las revistas } \\
\text { excluyendo otros tipos de documentos. Además en } \\
\text { esta investigación se ofrecía los hábitos de lectura. }\end{array}$ \\
\hline
\end{tabular}

Fuente: Elaboración propia

Precisamente los años posguerra eran los que mejor proveían un contexto para impulsar los estudios de usuarios. Era necesario potenciar el flujo de información científica y tecnológica que habidas cuenta catalizaba el progreso científico - técnico.

Desde los primeros estudios y hasta los realizados a mediados de los años 50, a partir del análisis de los investigaciones citadas en las obras de Siatri y Menzel se puede plantear que el objetivo principal de estos era conocer cuales eran los hábitos de lectura de los científicos, qué uso se hacía de la literatura científica y no para qué se usaba. Sin embargo ya a fines de esta década empieza a estar latente la necesidad de incorporar a estos estudios dimensiones relativas al aspecto emocional y afectivo de los individuos. Dos ejemplos se mencionan a continuación (Siatri, 1999):

Al observar las referencias bibliográficas incluidas por Mendel (1966: 45) de estudios de usuario del período 1964-1965 se pueden observar dos tendencias:

* Todos los estudios de usuarios están referidos exclusivamente a individuos científicos en el contexto de la comunicación científica.

* La mayor parte de las investigaciones es realizada a científicos de las llamadas ciencias duras, puras o naturales (bioquímicos, químicos, físicos, biólogos, ingenieros, médicos).

La necesidad de los sistemas de información de investigar acerca de la interacción del usuario con el sistema se ve potenciada por la influencia de la Teoría de Sistemas de Bertalanffy, la cual precisamente a finales de los años 40 se formaliza.

La ambición del sistemismo consiste en atender a la globalidad, a las interacciones entre los elementos más que a las causalidades (Mattelart, 1997).

Otro de los factores que influye en el aumento de los estudios de usuarios es el advenimiento de los servicios de diseminación de información y los índices permutados. Para los proveedores de servicios de información era importante la retroalimentación con sus usuarios.

Los estudios de usuarios al decir de Castillo (2003: 9) desde su arribo utilizaron técnicas bibliométricas. Ello en total correspondencia con el método científico imperante y el modelo de Ciencia dominante. La asunción de estos métodos cuantitativos era una manera de lograr el reconocimiento de la cientificidad de las investigaciones.

Al hablar sobre métodos de investigación se hace necesario recordar que en estos años, bajo el dominio del paradigma positivista y el cientificismo, las investigaciones adoptan el método cuantitativo, y los estudios de usuarios no constituyen excepción alguna.

Se puede comentar haciendo un análisis complementario de los trabajos de Menzel y Siatri que las principales técnicas e instrumentos utilizados fueron:

* Los cuestionarios

TABLA 2

Estudios donde se incorporan las dimensiones relativas a los aspectos emocionales y afectivo

\begin{tabular}{|l|l|}
\hline Herner(1954) & Examinó la confianza de científicos en la información técnica y su fuente de origen. \\
\hline Menzel(1958) & Estudió la influencia de la satisfacción del usuario en la adquisición de información.
\end{tabular}

Fuente: Elaboración propia 
* El análisis documental cuantitativo

* La observación

Sobre el método, técnicas e instrumentos de investigación es válido aclarar cómo desde el principio se demostró que el método cuantitativo no ofrece las respuesta a los problemas de investigación de los estudios de usuarios. Desde el estudio realizado por Bernal se obtuvo inconsistencia en los datos recopilados (Siatri, 1999). Al utilizar dos instrumentos de recopilación de datos (cuestionario y tarjetas donde diariamente registra lo que observaba) los resultados del análisis de los datos de uno no se correspondían con los del otro.

Muy relacionado a los estudios de usuarios aparece el término necesidad de información. Ya desde finales de los 50 y principios de los 60 este concepto se erige como concepto clave en los estudios de usuarios. Hacia 1958 Menzel realiza una investigación en el Bureau of Applied Social Research at Columbia University donde tiene entre sus principales objetivos la identificación de diferentes tipos de necesidades de información, Mote en 1962 para su examen creó 3 categorías de necesidades de información y Flowers en 1965 estudió las necesidades de información de físicos y químicos (Siatri, 1999).

Durante esta época se comienza a introducir teorías y metodologías desde las ciencias sociales y del comportamiento. Los resultados consistentes y detallados obtenidos en la serie de investigaciones realizadas por la American Psychological Association (APA) durante 1963 y 1969 contribuyen a que algunos, en realidad unos pocos, profesionales de la investigación dedicados a los estudios de usuarios comiencen a ponderar las posibilidades que ofrecen las técnicas y métodos propios de tipo de ciencias.

Alrededor de los años 70 en la literatura se habla de un esplendor de los estudios de usuarios. En esta década se extiende este tipo de estudios hacia otras comunidades de usuarios además de la científica. En este lapso los estudios de usuarios se orientan a examinar el uso de sistemas de información, su eficacia y eficiencia y cómo estas últimas pueden ser maximizadas (Siatri, 1999).

En estos años además se funda el Centre for Research on User Studies en la Universidad de Sheffield. De esta procede Wilson uno de los más conocidos y prolíferos que investiga y publica sobre Educación de Usuarios.

Como con anterioridad se expresó Belkin la define como estado anómalo de conocimiento $(1978,81)$. Por su parte Wilson (2002) sugiere la necesidad de definir el contexto de la necesidad pues reconoce que no solo existen factores internos, además hay diversos factores externos que influyen en el comportamiento de búsqueda de información (Siatri, 1999).

En los planteamientos de uno y otro autor se explicita a qué enfoque responde su formulación: el psico-sociológico.

Esta nueva variable que aparece en los estudios de usuarios surge acompañada de otras como comportamiento de búsqueda de información, comportamiento en el uso de la información.

En un estudio métrico realizado (Castillo, 2002: 36) se detectó que los autores con mayor producción sobre este tema en el período 1985-2001 de los trabajos registrados en LISA son Wilson, Meadows, Spink, Hernon, Line, Kantor, Saracevic, Slater. En ese mismo estudio se concluye que existen todavía pocas metodologías que abundan en el tema de las necesidades de información.

Sobre esta área se puede decir que el usuario ha sido y es estudiado con diferentes objetivos y metodologías. Estos estudios pueden asociarse a diferentes líneas de investigación correspondiente a temas como: Recuperación de información, Sistemas de información, Sociología de la información, Marketing de los servicios de información, entre otros.

Almeida (apud Hewins, 1990) refiere que existen tres nuevas formas de abordar los estudios de usuarios:

* El valor del usuario, enfocado en las percepciones de utilidad y valor de los sistemas de información;

* Sense-making, que examina la forma cómo las personas "dan sentido" a sus mundos y cómo la información es utilizada en este proceso y

* Los estados anómalos de conocimiento (ASK), que examina cómo las personas buscan información en situaciones en que su conocimiento es incompleto.

A pesar que la autora afirma que estos tres enfoques constituyen paradigmas se puede comentar, en opinión de la autora que suscribe el presente artículo, que estos tres enfoques no alcanzan tal categoría. Son formulaciones resultado de la influencia de un paradigma que se ha introducido en las disciplinas informativas desde los años 70: el cognitivismo.

En correspondencia con los tres enfoques antes mencionados se pueden encontrar varias clases de estudios de usuarios como estudios de necesidades de información, sobre el 
comportamiento informacional, sobre la aceptación de tecnología de información y otros.

Sobre los dos primeros se ha hecho referencia en algún que otro momento a lo largo del trabajo por ser las dos clases pioneras de esta área. Sin embargo las transformaciones operadas por las tecnologías en el dominio informacional crean la necesidad de incorporar una nueva clase: los estudios de aceptación de las nuevas tecnologías devenidos más tarde en los estudios del consumidor de información en la Web.

Los estudios relativos a las tecnologías de la información se han nutrido de diversas teorías. Entre ellas se pueden mencionar la Teoría de Difusión de la Innovación, la Teoría de la Acción Racional, la teoría del Comportamiento Planeado, la Teoría de la Acción Comunicativa. En estos estudios se incorporan nuevas variables como usabilidad y amigabilidad, además de mantener otras sociológicas, demográficas y psicológicas. Otras teorías que se utilizan en este tipo de estudios son las Socio-Técnicas, "las cuales se aplican sobre todo para el estudio de las tecnología dentro de las organizaciones" (Almeida, 1999).

Dentro de la tipología de estudios de usuarios se encuentra además los Estudios sobre la calidad de uso de la información. Vinculado directamente a la introducción de la filosofía de la Calidad Total que al ser adaptada a los sistemas de información se divide en tres dimensiones fundamentales (Erickson y Törn, 1997):

* Calidad asociada al uso de la información

* Calidad asociada al Negocio

* Calidad asociada al trabajo del sistema de información

Lindroos (1997) en su investigación encontró varios modelos relativos a la calidad de los sistemas de información en ambiente Web, de ellos existe uno dedicado precisamente a la satisfacción informacional del usuario. Este modelo está desarrollado sobre la base de la Teoría de Sistemas. Su principal mérito es considerar las expectivas del usuario un factor clave, para el logro de la satisfacción informacional, que está formado por las características del usuario y su organización.

\section{¿METATEORÍA O TEORÍA? ASOCIADA AL ESTUDIO DE USUARIOS DE LA INFORMACIÓN}

Hasta este momento se han expuesto varias aristas que están directamente vinculadas al estudio del usuario.
También al inicio del presente se mencionaron algunas teorías que provenientes de diversos campos han influido en la concepción de la noción usuario. Sin embargo aún cuando se ha hecho mención sobre algunas teorías que han impactado esta área de investigación aún queda, a juicio de la autora del presente trabajo, por presentar si existe alguna teoría o metateoría que haya sido desarrollada para abordar los diferentes estudios de usuarios.

En las diferentes ramas del saber, sus disciplinas y sus áreas de investigación es recurrente escuchar hablar sobre las teorías, aunque sin embargo algunos campos utilizan el término metateoría. Cuáles son las principales diferencias entre teoría y metateoría.

Existen diversas concepciones de lo que es una teoría, una de ellas la considera un conjunto de conceptos relacionados que pretenden representar la naturaleza de una realidad. Para Kerlinger "Una teoría es un conjunto de constructos (conceptos) interrelacionados, definiciones y proposiciones que presentan un punto de vista sistemático de los fenómenos mediante la especificación de relaciones entre variables, con el propósito de explicar y predecir los fenómenos" (1988:10). Es importante señalar que en una teoría, es tan importante el poder predictivo y el control que ésta tenga, como su capacidad para explicar y ayudar a comprender el fenómeno.

Una teoría en Ciencias de la información es "una explicación teórica de la eficacia de los sistemas de información, del comportamiento de los usuarios, de la función de los diferentes elementos de búsqueda" (Hjorland, 1998). Es el propio Hjorland quién establece que algunos enfoques específicos no deben ser considerados como teorías y por tanto los enfoques aportados por Kuhlthau, Wilson, entre otros no deben ser considerados como teorías.

Pero ciertamente aun cuando alguien se aventura a reflexionar sobre la teoría, como área de conocimiento, debe recordar que paradigmáticamente el modelo y los supuestos que más desarrollados están, han sido elaborado desde "las ciencias duras".

Uno de los supuestos fundamentales propuestos desde las "ciencias duras" es que la teoría debe proveer un conjunto de leyes que deben ser rigurosamente constatables por la evidencia empírica y que mientras la teoría no está comprobada ni ha manifestado su capacidad explicativa, sigue siendo una hipótesis (Buckland, 1999: 34; Abarca, 1996).

¿Son válidos estos para las ciencias humanas y del comportamiento, para las Ciencias de la Información? Dada la diversidad de factores que intervienen en las situaciones donde se realizan estudios humanos y del 


\section{Yohannis Martin-Lahera}

comportamiento ise puede esperar que rigurosamente se cumplan las leyes que enuncia una teoría X?; o cúales son los indicadores que establecen que los postulados de los científicos de este dominio han dejado de ser hipótesis y se han convertido en teorías si al decir del propio Buckland en este dominio la percepción juega un papel fundamental en la valoración y aceptación de una teoría.

De acuerdo a la clasificación metateórica propuesta por Abarca*(1996) de las teorías científicas se puede inferir que las teorías relativas a los estudios de usuario serán cuasiimperfectas y hasta imperfecta en la mayoría de los casos.

Las teorías de cualquier dominio pueden convertirse en objeto de estudio, y sobre ella se pueden elaborar, a su vez, constructos mentales. Así aparece un término que posee dos acepciones metateoría. Una de las acepciones es como disciplina que se encarga de estudiar las propiedades de la teoría científica; la otra es como teoría cuyo objeto de estudio es teoría. Ello implica que una metateoría es un conjunto de asunciones más amplias y menos específicas que la teoría.

Se plantea que esas asunciones están conectadas a puntos de vista filosóficos, y que generalmente son parte de la interdisciplinariedad (Hjorland, 2000).

Además se explica que la metateoría solo tiene razón de ser, sentido cuando la teoría o teorías por sí solas no alcanzan para entender el fenómeno. De acuerdo a Morea se pueden encontrar tres variedades de Metateorización (1997):

* Metateorización como medio para obtener una comprensión mas profunda de la teoría.

* Metateoría como preludio para la realización del desarrollo de esta teoría.

* Metateorización como fuente de las perspectivas que sostiene toda la teoría de un dominio.

Al decir de Hjorland (1998) los enfoques metateóricos más importantes en las Ciencias de la información son

\footnotetext{
* Teorías perfectas: Una teoría es perfecta si cumple las siguientes propiedades: 1) consistencia (libre de toda contradicción); 2) compleción (todas las fórmulas verdaderas de la teoría pueden derivarse como teoremas de teoría); 3) decidibilidad (existe un método infalible para saber si un teorema de teoría es o no derivable dentro de la teoría); 4) categoricidad (todas las interpretaciones de las fórmulas de teoría tienen la misma estructura). Teorías cuasiperfectas: las que poseen las dos primeras cualidades de las perfectas: consistentes y completas. Teorías cuasiimperfectas: las que son Teorías imperfectas consistentes, pero no son ni completas ni decidibles.: las que no tienen ninguna de las cuatro propiedades mencionadas.
}

el "paradigma físico" y el "enfoque cognitivo". El mismo afirma que en este dominio faltan "buenas teorías" que la mayoría de las que sustentan los postulados no se consideran propias sino de otras disciplinas.

La realidad discursiva teórica de los estudios de usuarios ha sido caracterizada caótica por la plétora de conceptos, teorías, enfoques, métodos y descubrimientos de la que está plagada (Dervin, 2003; Hojrland, 1998).

Este ha sido creado por la producción aislada - y en la mayoría de los casos - desarraigada del estado general de desarrollo del área de investigación (Dervin, 2003).

Ello provoca de acuerdo con Dervin contradicciones e inconmensurabilidad entre los investigadores que usan un conjunto de conceptos, variables, métodos o teorías, y aquellos que usan otro. Pero este fenómeno afirma que no solo se da al interior de la "comunidad discursiva", se extrapola y ocurre entre aquello que desde diferentes disciplinas persiguen el mismo asunto y llegan a resultados radicalmente opuestos.

Sin embargo apunta que lo más irónico de la situación es que precisamente quienes intentan construir el consenso, constituyen parte del problema, y explica que se incluyen en el problema por dos razones fundamentales:

* La mayoría de los trabajos y recompensas que reciben los investigadores provienen de las críticas y cambios hechos al conjunto de prácticas postuladas por otros,

* La existencia de fuerzas ocultas de tipo económico, político, cultural, etc. que subyacen en el trabajo y que propician el aislamiento y las discrepancias.

No obstante, se hace mención de otra ironía: la mayoría de los múltiples enfoques que existen e intentan crear el consenso están anclados en la misma pluralidad de fuerzas y teorías que han llevado al dilema.

¿Significaría lo anterior que todos los estudiosos deben acogerse a un único enfoque, a una única propuesta y abandonar su visión? La misma autora advierte que no se trata de abandonar los caminos elegidos y/o construidos que se han experimentado. La idea es se sigan desarrollando pero profundizando en las diferencias y aumentar el aislamiento y la contradicción sino que se siga produciendo más pero con una visión mucho más amplia.

Supondría lo anterior, a juicio de la que suscribe el presente trabajo, llegar espontáneamente a puntos comunes, de encuentro que enriquecerían el desarrollo de este dominio, solventaría el caos y crearían entre otras la necesidad 
de colaboración entre los miembros de la comunidad discursiva. En otras palabras se requiere la existencia de asunciones metateóricas que sirvan de base y sustento a la pluralidad de enfoques.

Mas ipor qué hablar de metateorías en el dominio Usuario? Al decir de Hjorland frente a la inexistencia de teorías sólidas en algunos dominio se ha experimentado el desarrollo de metateorías (aproximaciones teóricas más amplias y menos específicas) que derivan en trabajos teórico-empírico.

Un ejemplo claro de metateoría es el conjunto de aproximaciones realizado por Brenda Dervin, denominado Sense-Making. Este enfoque comenzó a ser desarrollado en los años 70, aunque no fue hasta 1983 que la denominación se usó (Dervin, 2003: 137).

Al principio, la propia Dervin así lo declara, el SenseMaking fue desarrollado como un enfoque para estudiar desde una perspectiva comunicativo las necesidades de información, la búsqueda y el uso de la información. Por ello se plantea que esta metateoría descansa en el reconocimiento de la naturaleza de la comunicación humana. A partir de este reconocimiento la autora concluye que los modelos dominantes usados en la comunicación formal, la educación y los sistemas de información no son efectivos ni eficientes porque están basados más en el transporte/trasmisión de información (algunas veces llamado "banking”) que en la comunicación. Por ello Dervin afirma que el Sense-Making es una metodología diseñada para el estudio comunicativo de la comunicación.

La creadora de este enfoque se siente deudora (Dervin, 1999), y por tanto reconoce que en su propuesta hay una fuerte influencia, de R.F. Carter. Para ella de los trabajos de este último lo que mayor impacto tuvo en el SenseMaking fueron sus aportes teóricos que se han enfocado en el desarrollo de los enfoque comunicativos al estudio de la comunicación. Esta autora alude a otros autores que tributan como Bourdieu, Bruner, Freire, Gadamer, Habernas, McGuire, Morrow, Murdock.

Ahora bien, ipor qué considerar el Sense-Making una metateoría? Dervin (2003:146) es quién precisamente considera que este enfoque es una metateoría. Al sustentar su posición la autora ejecuta varias estrategias una de ellas es ofrecer la definición de metateoría como aquel conjunto de "presupocisiciones que proveen perspectivas generales o maneras de buscarlas, basadas en asunciones acerca de la naturaleza de la realidad y los seres humanos (ontología), la naturaleza de la cognición (epistemología), los propósitos de la teoría e investigación (teleología), los valores y la ética (axiología) y la naturaleza del poder (ideología)".

Las asunciones del Sense-Making sobre la naturaleza del ser humano están necesariamente referidas a la naturaleza de la realidad, y la naturaleza del acto de conocer. Por su carácter genérico, porque sobrepasa los límites de metodología y método es que se considera metateoría. La propia Dervin en su página personal sostiene que quienes intenten acercarse y aplicar el Sense- Making lo que deben hacer es acercarse a los trabajos teóricos que existen al respecto, pues las aplicaciones no le permitirán una comprensión cabal del enfoque.

Dervin afirma que se pueden mencionar numerosos casos de usos y malos usos del Sense-Making. Plantea que algunas personas usaron el Sense-Making primeramente como un método, dejando a un lado sus intenciones metateóricas, haciendo alguna referencia a ella como teoría sustantiva y tomando de ella lo que les parecía adecuado para su estudio; otras han extrapolado desde su enfoque metateórico para teorizar y estudiar una variedad de fenómenos en diversos géneros de investigación. Estos últimos, apunta, no incluyen solo los estudios en Bibliotecología y Ciencias de la Información, sino también a los de Periodismo, Culturología, Educación, Pedagogía, entre otros, siempre asociados a la comunicación.

Más allá de la metateoría de Dervin existen varias teorías que intentan explicar el comportamiento informacional. Las teorías psicológicas acerca de los usuarios, su comportamiento y su cognición juegan un rol fundamental en las Ciencias de la información (Hjorland, 1998). Algunas teorías psicológicas son el constructivismo y el cognitivismo, ellas aparecen explicadas en muchos trabajos, bajo el paraguas cognitivo por ejemplo aparecen Wilson, Davis, Córdova, Bonnie-Wai-Yi, Cheuk, entre otros. Aunque las más difundidas son las provenientes de la Psicología existen otras que fundamentalmente provienen del campo comunicacional.

Case (2002) cita algunas teorías que merecen ser mencionadas como el Principio del Menor Esfuerzo, MASA, Uso y Gratificaciones, y del Entretenimiento.

Principio de Zipf o del Menor Esfuerzo: Este principio está indisolublemente ligado a la Ley de Zipf, que ha sido incorporada dentro de las leyes informétricas. A pesar de tener gran aceptación la Ley de Zipf que explica el cómo ocurre, este principio que intenta explicar el por qué, no ha tenido muchos seguidores al decir de Buckland (1999). La base del principio de George Zipf descansa en el supuesto que cualquier individuo traza un curso de acción en el 
que desarrolla un conjunto de acciones que selecciona a partir del criterio: el menor esfuerzo requerido.

En términos de búsqueda de información es aquí donde se aplica la regla 80-20 (las personas utilizan solo un $20 \%$ del total de fuentes de información disponibles para resolver el $80 \%$ de sus necesidades por la propensión humana de solucionar sus necesidades utilizando los mismos recursos informativos que en otros momentos ha utilizado). Otra conclusión referida al comportamiento en la búsqueda de información derivada de estos estudios es que las personas tienden a priorizar el criterio fácil accesibilidad antes que el de alta calidad en el momento de seleccionar la información. Es decir, que en el proceso de búsqueda de información las personas decidirán usar un recurso basado en su facilidad de acceso más que en la confiabilidad de la información que podría contener.

Recientemente se ha realizado una investigación sobre la Web y este principio donde se explican un conjunto de elementos que podrían argumentar con mayor cantidad de elementos los párrafos anteriores. Este trabajo fue desarrollado por Baeza.

Usos y Gratificaciones: En esta teoría las personas son vistas como selectores activos de los recursos de información porque realizan procesos de interpretación sobre la información que recuperan. Los usuarios se consideran como receptores activos de información. En esta teoría el foco de atención está en qué recursos hacen para las personas en lugar de preguntarse qué le hacen los recursos a las personas. El elemento fundamental de la hipótesis de esta teoría es relacionar consumo, uso y efecto de los media con la estructura de necesidades que caracteriza al destinatario. Intenta explicar el consumo de los efectos de los medios en función de las motivaciones y de las ventajas que extrae el usuario.

La conexión entre satisfacción de la necesidad y elección del recurso está representada como una opción del usuario en un proceso racional de adecuación de los recursos disponibles a los fines perseguidos.

Uso Mediático como Acción Social (Masa): En esta teoría la información es vista como objeto, en el ambiente social, que requieren de la interpretación por parte de los individuos. Cuán significativo es un objeto de información, sólo puede ser determinado por todos los otros factores que existen en el ambiente que rodea al objeto y al individuo, más todos los factores internos de este último (ejemplo personalidad). Basada en aportes como el de Fish, Jauss, entre otros Lindloff en esta teoría inserta un concepto "comunidad interpretativa".
Así Orozco (citado por Varela 2000) define una comunidad de interpretación se entiende básicamente como un conjunto de sujetos sociales unidos por un ámbito de significación del cual emerge una significación especial para su actuación social (agency).

La definición de una comunidad interpretativa remite al debate acerca de la subjetividad, libertad y límites del individuo en el acto de lectura. Estas comunidades interpretativas, más que el texto o el receptor, son las que producen el sentido y quienes son responsables por la emergencia de rasgos formales. Las comunidades interpretativas están integradas por aquellos que comparten estrategias interpretativas no para leer sino para escribir textos, para constituir sus propiedades, en otras palabras, estas estrategias existen previamente al acto de leer y en consecuencia determinan la forma de lo que se lee.

Esta propuesta supone una traslación del texto al contexto y pone de relieve la relación entre consumo e identidades, así como la tendencia a la constitución de identidades nómadas y la fragmentación social y cultural. Quizás por ello Varela(2000) afirma que los análisis de la década del 90 tendieron crecientemente a plantear los modos de consumo de las comunidades interpretativas de consumidores.

Teoría del entretenimiento: Esta teoría plantea que la mayoría de las piezas de información y nuestro involucramiento con ellos acarrean aspectos relativos tanto al trabajo como al recreo. El aspecto más importante que postula esta teoría es que, incluso cuando se interactúa con la información puramente por razones de trabajo, las personas buscan y seleccionan aquellos recursos que sean amenos por su estilo o formato.

No obstante, a pesar de todo lo apuntado anteriormente se puede afirmar que al decir de Hjorland (2000) una teoría general sobre el comportamiento en la búsqueda de información debe incluir una arista evolucionaria, donde se considere cómo los organismos han adaptado su aparato cognitivo a las demandas surgidas para poder estar a tono con su ambiente.

A juicio del propio autor ella debe describir estados cualitativos en la evolución antes mencionada y debe además explicar la naturaleza teolológica u orientada por objetivos del comportamiento de los organismos vivos.

Más adelante en su trabajo expresa que como teoría debe definir las características esenciales en la búsqueda de información por parte de los humanos, incluyendo una descripción de sus determinantes humanos y sociales. 


\section{¿Teoría o metateoría? En el dominio usuario}

Esta teoría debe considerar los costos y beneficios de la búsqueda de información y los mecanismos sociales que influyen en su valor.

Adicionalmente, Hjorland expresa que tal teoría debe explicar una serie de factores como la utilización de los diferentes tipos de recursos de información, las estrategias que los usuarios usan y su eficacia, entre otros. Para él una teoría general debe formular que es lo esencial, cuáles son los factores críticos, cuáles pueden ser generalizadas desde los diferentes dominios, y cuáles están afectando la eficacia de la búsqueda de información. Tales factores, al decir del mismo, están referidos a las percepciones básicas que las personas tienen del mundo y a sus asunciones epistemológicas fundamentales.

Hasta aquí se ha hecho una primera aproximación a este aspecto, sin embargo este ha de ser el primer intento que necesariamente conlleve a que otros profundicen sobre este tema, y aborden con exhaustividad no solo la metateoría de Dervin sino también los enfoques de Wilson, Ingwersen y Kuhlthau. Un posterior trabajo sobre este tema develaría muchas incógnitas es cierto pero también es cierto que llevaría a encontrar el rumbo por el cual se elige seguir y a entender por cuál o cuáles hemos estado transitando.

\section{EPÍLOGO}

Al comenzar el trabajo se partía de la reflexión sobre el par usuario-sistema. Entonces se aportaban elementos que sustentan la idea que entre el usuario y el sistema de información no existen fronteras, sino puntos de encuentro donde hay intercambio benéfico y necesario para el desarrollo mutuo, donde se adquieren los elementos para identificar a los componentes del sistema.

Dicha reflexión necesariamente está influenciada por las líneas de pensamiento dominantes de la época. Cada etapa generalmente produce desde el punto de vista intelectual planteamientos coherentes con los discursos dominantes. Este texto así ha tratado de reflejar variaciones sufridas en la concepción, comprensión e investigación del usuario en el dominio informacional.

Los caminos tomados por los estudiosos de esta área han estado profundamente marcados por las concepciones filosóficas dominantes durante la época de su producción, los supuestos, postulados, teorías de la ciencia en la cual se formaron y los de las diferentes disciplinas informativas. Así en la dimensión usuarios del mundo informacional se han identificado como enfoques dominantes el conductismo y el cognitivismo.
El punto de vista cognitivista que impera desde la década del sesenta aboga por una aprehensión de la noción usuario de forma individualizada.

"Tanto el conductismo como el cognitivismo son,... demasiado positivistas racionalistas... Los usuarios se ven como mecanismos abstractos, aislados de la esfera sociocultural; no como mecanismos adaptativos, integrados estrechamente en nichos específicos" (Hjorland, 1995).

En la actualidad se aboga por un enfoque sociocognitivista que cuenta con Hjorland entre sus defensores. Esta tendencia se opone a la idea de concebir "el contexto disciplinario como parte de la estructura cognoscitiva de un individuo" (Hjorland,1995), propugna que el usuario individual sea entendido en el contexto de su disciplina. Por ello de los planteamientos de Hjorland y Frohmann (1992) se desprende que el estudio y la comprensión de la noción usuario debe efectuarse a partir de posturas socioculturales que permitan construir principios reflejo de la realidad objetiva y no de la percepción subjetiva que tienen los usuarios del mundo de la información.

Si los usuarios son quienes justifican la razón de ser de estos, entonces los profesionales vinculados a la investigación y praxis en el mundo informacional deben crear propuestas acorde con las nuevas visiones de los seres humanos y del conocimiento. Visiones que apuntan hacia enfoques como el planteado por Hjorland "más orgánicos, contextuales, socioculturales y de lo específico del dominio".

Artigo recebido em 19-11-2004 e aceito para publicação em 29-03-2005.

\section{REFERENCIAS}

ABARCA FERNÁNDEZ,R. R. Vocabulario filosófico científico. Arequipa : Universidad Católica de Santa María, 1996. Disponível em: <http://www. ucsm.edu.pe/rabarcaf/vofici04.htm>. Acesso em: 12 maio 2004.

ALMEIDA FURQUIM, T. de Perspectiva para o estudo do consumidor de informaçao na web. Revista de Biblioteconomia de Brasília, Brasília, v. 23/24, n. 1, p. 119-131, 1999-2000.

ALONSO, M. Una aproximación interdisciplinar al estudio del usuario de la información: bases conceptuales y metodológicas. Investigación Biliotecológica, v. 13, n. 26, p. 112-124, 1999.

BAEZA, R. La web y el principio del mínimo esfuerzo. Disponível em: $<$ http://www.dcc.uchile.cl/ rbaeza/inf/zipf.htm>. Acesso em: 13 jun. 2004.

BELKIN, N. Information science and the phenomena of information. Journal of American Society for Information Science, v. 27, p. 197-204, 1978.

BUCKLAND, M. Library services in theory and context. Berkeley : Berkeley Digital Library SunSITE, 1999. 146 p. 


\section{Yohannis Martin-Lahera}

CASE, Donal O. Looking for information: a survey for research on information seeking, needs and behavior. Disponível em: <http:// wmgpromotionalgifts.co.uk/Case-Looking-for-Information-924-672-219. X.html>. Acesso em: 20 maio 2004.

CASTILLO ZAYAS, M. del. Usuarios de alto rendimiento deportivo de lucha y béisbol: sus necesidades de información. 2002. Tesse (Licenciatura em Bibliotecología) - Universidad de la Habana, La Habana, 2002.

DERVIN, B. On studying information seeking methodologically: the implications of connecting metatheory to method. Information Processing and Management, v. 35, p. 727-750, 1999.

. Sense-making's journey from metatheory to methodology to method: an example using information seeking and use as research focus. In: ; FOREMAN-WERNET, L. Sense-making methodology reader: selected writings of Brenda Dervin. Cresskill, NJ : Hampton, 2003. p. 133-164

ERIKSSON, I.; TÖRN, A. Introduction to IST. Information and Software Technology, v. 39, n. 12, p. 797-799, Dec. 1997. Special issue on Information System Quality.

FERNÁNDEZ MOLINA, J. C.; MOYA-AREGÓN, F. Perspectiva epistemológica humanas en la documentación. Revista Española de Documentación Científica, v. 25, n. 3, p. 145-167, 2002.

FROHMANN, B. The power of images: a discourse analysis of the cognitive viewpoint. Journal of Documentation, v. 48, p. 365-386, 1992.

HEWINS, E. Information need and use studies. Annual Review of Information Science and Technology, v. 25, p. 145-172, 1990.

HJORLAND, B. Toward a new horizont in information science: domain analysis. Journal of American Society for Information Science, v. 46, n. 6, p. 400-425, 1995.

Theory and metatheory of information science: a new interpretation. Journal of Documentation, v. 54, n. 5, 1998.

. Information seeking behavior: what should a general theory look like? New Review of Information BehaviourRresearch: Studies of Information Seeking in Context. v. 1, p. 19-33, 2000.
KERLINGER, F. N. Investigación del comportamiento. México : McGrawHill, 1989.

LINARES, R. La ciencia de la información y sus matrices teóricas: contribución a su historia. 2003. Tesse (Doctorado en Ciencias de la Información) Universidad de la Habana, La Habana, 2003.

LINDROOS, K. Use quality and the world wide web. Information and Software Tecnology, v. 39, n. 12, p. 827-836, 1997.

MATTELART, A.; MATTELART, M. Historia de las teorías de la comunicación. Barcelona : Paidós, 1997. 142 p.

MEDINA, I. Desde el otro lado. La Habana : Pablo de la Torriente, 1995.

MENZEL, H. Information needs and uses in science and technology. Annual Review of Information Science and Technology, v. 1, p. 41-69, 1966.

MOREA, L. Metateoría y sociología contemporánea. Disponível em: $<$ http://www.monografias.com/trabajos11/metateo/metateo.shtml>. Acesso em: 14 jun. 2004.

NÚÑ̃Z PAULA, I. A. Enfoque teórico-metodológico para la determinación dinámica de las necesidades que deben atender los sistemas de información en las organizaciones o comunidades. 2003. Tesse (Doctorado en Ciencias de la Información) - Universidad de la Habana, La Habana, 2003.

SANZ CASADO, E. Manual de estudios de usuarios. Madrid : Pirámide, 1994. $288 \mathrm{p}$.

SIATRI, R. The evolution of user studies. Libri, v. 49, n. 3, p. 132-141, 1999.

VARELA, M. De las culturas populares a las comunidades interpretativas. Disponível em: <http://www.innovarium.com/CulturaPopular/culturas populares.pdf $>$. Acesso em: 2 jun. 2004.

WILSON, T. D. Recent trends in user studies: action research and qualitative methods. Information und Dokumentation MIB, v. 11, n. 80, p. 32-56, 2002. Disponível em: <http://informationr.net/ir/paper76.html>. Acesso em: 20 maio 2004. 